\title{
Faktor-Xa-Inhibitor auch in der Sekundärprävention wirksam
}

- Vitamin-K-Antagonisten verhindern bei Vorhofflimmern effektiv Schlaganfälle. Allerdings steht nach Prof. Werner Hacke, Heidelberg, dieser Wirksamkeit - neben mühsamer Einstellung, kontinuierlicher Überwachung und Interaktionen - ein erhöhtes Risiko für intrakranielle Blutungen entgegen.

Als neue Therapieoption für die Antikoagulation bei Vorhofflimmern stellte Hacke den direkten, einmal täglich oral einzunehmenden Faktor-Xa-Inhibitor Rivaroxaban (Xarelto ${ }^{\oplus}$ ) vor. Dass er in der Primärprävention von Schlaganfällen und systemischen Embolien bei hohem Schlaganfallrisiko (mittlerer $\mathrm{CHADS}_{2}$-Score etwa 3,5) Warfarin nicht unterlegen ist, ergab die randomisier- te, doppelblinde Studie ROCKET AF (Ereignisrate 2,12 vs. 2,42 pro 100 Personenjahre). Nahmen die Patienten die Studienmedikation ein, war der Faktor-Xa-Inhibitor dem Vitamin-K-Agonisten signifikant überlegen (Ereignisrate 1,71 vs. 2,16; $p<0,001$ ).

Insgesamt 14264 Patienten mit Vorhofflimmern wurden in ROCKET AF entweder mit Rivaroxaban (1 $x$ täglich $20 \mathrm{mg}$ ) oder Warfarin (Ziel-INR 2-3) behandelt. Jeder zweite Patient hatte bereits eine TIA, einen Schlaganfall oder eine systemische Embolie in der Vorgeschichte.

Hacke stellte eine Subanalyse zur Sekundärprophylaxe vor, in der die Daten der Patienten mit vorherigem Schlaganfall oder sys- temischer Embolie ausgewertet wurden ( $\mathrm{n}=$ 7468). Sie bestätigte die Ergebnisse der Gesamtstudie: Sowohl die Rate der Ereignisse $(2,26$ vs. 2,60$)$ als auch der Blutungen $(13,31$ vs. 13,87$)$ war vergleichbar, die Raten tödlicher und intrakranieller Blutungen waren unter dem Faktor-Xa-Inhibitor nummerisch geringer $(0,59$ vs. 0,80$)$. Insgesamt sei der Effekt von Rivaroxaban im Vergleich zu Warfarin in der Sekundärprophylaxe tendenziell besser und in der Primärprävention mindestens vergleichbar, resümierte Hacke.

- Andrea Warpakowski

Quelle: Pressekonferenz „Neue Dimensionen der Antikoagulation“, Hamburg, Mai 2011 (Veranstalter: Bayer Health Care)

\section{Chronische Obstipation}

\section{Nicht nur eine Folge des westlichen Lebensstils}

- Eine chronische Obstipation ist nicht allein Folge des westlichen Lebensstils und nur selten durch ein intra- oder extraluminales Hindernis zu erklären, betonte Priv.-Doz. Jutta Keller, Hamburg. Meist hat die anhaltende Obstipation eine funktionelle Ursache. Problematisch ist die "Slow transit Constipation", die häufig nicht auf diätetische Maßnahmen wie Trockenpflaumen, Milchzucker und vermehrte körperliche Bewegung und die zusätzliche Laxanziengabe als zweite Stufe der Obstipationstherapie anspricht. Besondere Probleme bereitet auch eine chronische Obstipation bei neurologischen Grunderkrankungen, z. B. bei autonomer Neuropathie bei Diabetes. Bei Parkinson kann sie sogar das erste Symptom sein. Auch obstipierende Medikamente wie Morphine machen Lebensstiländerungen und die Gabe von Laxanzien notwendig.

\section{Prokinetikum Teil der Stufentherapie} Greifen diätetische Maßnahmen und Laxanzien nicht, stellt das Prokinetikum Prucaloprid (Resolor ${ }^{\circledast}$ ) die dritte Stufe der Therapie der chronischen Obstipation dar, be-

\section{Abbildung 1}

Prucaloprid bei schwerer, konventionell refraktärer Obstipation (Zulassungsstudien)

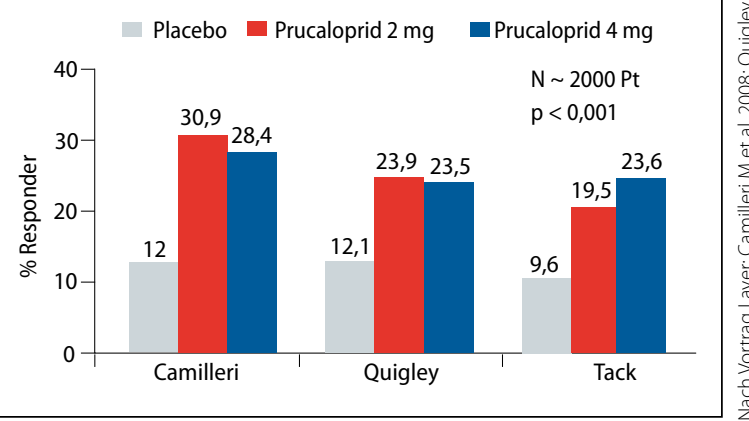

Abb. 1 Prucaloprid: Eine Chance bei schwerer, konventionell therapierefraktärer Obstipation.

richtete Prof. Peter Layer, Hamburg. Der 5- $\mathrm{HT}_{4}$-Agonist ist zugelassen für die symptomatische Behandlung der chronischen Obstipation bei Frauen, bei denen Laxanzien nicht adäquat zu einer Besserung führten. Die Beschränkung auf Frauen ist durch die Geschlechterverteilung in den Zulassungsstudien bedingt; eine Männerstudie läuft, so Layer.

Die Ansprechraten in Studien lagen bei der Standarddosierung von 2 mg Pruca- loprid bei schwerer, konventionell refraktärer Obstipation zwischen 19,5 und $30,9 \%$ (Abb. 1). Die reduzierte Dosis von $1 \mathrm{mg}$ bei über 65-Jährigen oder bei eingeschränkter Nierenfunktion war ähnlich wirksam wie die Standarddosis. Die Patienten waren auch in der offenen Studienverlängerung meist mit der Therapie zufrieden. Nur 5\% hatten wegen gastrointestinaler Nebenwirkungen oder Kopfschmerzen die Behandlung abgebrochen. Layer betonte, dass solche Beschwerden v. a. am ersten Tag auftreten. Kardiale unerwünschte Ereignisse wie bei dem verschiedene Rezeptoren beeinflussenden Cisaprid sind bei Prucaloprid laut Prof. Michael Schemann, München, nicht zu erwarten, da die Substanz hoch selektiv für den 5 - $\mathrm{HT}_{4}$-Rezeptor ist.

- Friederike Klein

Quelle: Satellitensymposium „Prucaloprid: Präzisionswerkzeug am Serotoninrezeptor", DGIMKongress, Wiesbaden, Mai 2011 (Veranstalter: Shire Deutschland) 